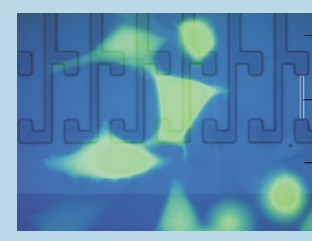

The creation of a viable biosensor using living cells and semiconductor devices has been thwarted by a variety of technical glitches. In theory, cells can be attached close to the "gate" region of field-effect transistors, which generate currents in the presence of small local voltage changes. However, cultured cells keep their distance both from one another and from their supportive substrate, limiting the size of the measurable voltage differential. In this issue, Peter Fromherz and his colleagues (see p. 121) partially overcome this particular problem. They transfected HEK293 cells with recombinant maxi-K channels, and then grew these on a silicon-coated, field-effect transistor. The large currents mediated by these channels created a voltage differential sufficiently large to be detected by the transistor. Most intriguing, the ion channels became concentrated at the cellsilicon interface, suggesting that further molecular manipulations to enhance the density of channels at the "gate" might enhance the signal-to-noise ratio of this device (see also p. 114).

$L F$

\section{Cutting out plant markers}

One of the biggest barriers to public acceptance of transgenic crops has been the presence in those crops of antibiotic marker genes. Previously developed systems to generate marker-free transgenic plants were either unreliable or not applicable to crops propagated by seeds (i.e., most crop plants). On page 157, Zuo et al. develop a chemical-inducible DNA excision system in transgenic Arabidopsis plants. They introduced into the Arabidopsis genome the Cre recombinase gene and the marker gene flanked by loxP recombination sites. They then induced the recombinase gene with $\beta$-estradiol to eliminate both itself and the linked marker DNA from the genome. The induction system shows remarkably tight control and high recombination efficiency (see also p. 115).

This month in Nature Biotechnology written by Liz Fletcher, Judy Jamison, and Meeghan Sinclair.
Studies have demonstrated the role of $\mathrm{CD} 4 \mathrm{~T}$ cells in allergic and autoimmune disease, but the search for a means to selectively target and eliminate the cells responsible has proved difficult. On page 142, Casares et al. describe a method for
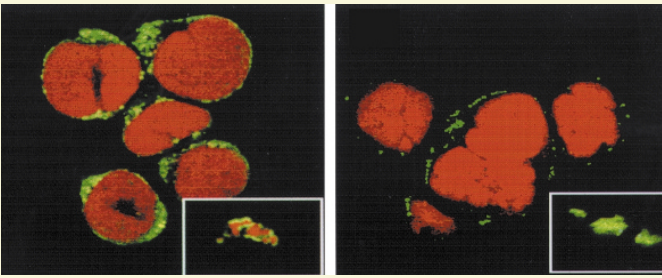
delivering drugs to antigen-specific $\mathrm{T}$ cells. They engineered a construct to target and destroy specific $\mathrm{T}$ cells by attaching the drug doxorubicin to recombinant MHC class II-peptide chimeras on an immunoglobulin scaffold. They demonstrated that the construct could bind to and deliver the drug to $\mathrm{T}$ cells that specifically recognized the peptide-MHC complex, thereby killing the cells. Delivery of the construct in transgenic mice reduced the frequency and proliferation of T cells specific for the peptide-MHC complex. The work has potential application for drug delivery to specific pathogenic $\mathrm{T}$ cells.

MS

\section{Engineering softer rice}

Wheat grain texture is critical to end-product quality, with direct effects on milling and baking quality, and producers have exploited the natural hardness or softness of wheat kernels to fill different market niches. The almost invariably hard texture of cereal grains outside the Triticeae tribe (e.g., rice and corn), however, has limited their usefulness and quality both in foods and in other products. On page 162, Krishnamurthy and Giroux describe the production of transgenic rice (Oryzae sati$v a$ L.) with softer kernels. Their strategy relied on the observed correlation between softer textured wheat, oats, and barley and the presence of two lipid-binding proteins, puroindolines $\mathrm{a}$ and $\mathrm{b}$. By inserting the pin $A$ and $\operatorname{pin} B$ genes into rice and assessing their effect on cereal grain texture, they showed that grain hardness was reduced in transgenic rice plants expressing PINA and PINB. Flour milled from the softer grains had reduced starch damage and an increased percentage of fine flour particles.

\section{Nucleic acid mutation detector}

Strategies to detect small genetic differences have employed DNA ligase enzymes in sequence detection because the enzymes are very sensitive to base mismatches at the ligation junction, but the enzymes show low activity with RNA and have other limitations. On page 148, Xu et al. describe the coupling of fluorescent reporter probes in a nonenzymatic, phosphorothioate-iodide ligation reaction for mutation detection. They demonstrate that the ligation reaction is able to detect sequences on both DNA and RNA templates, and that it works well in different formats such as in solution or on gels. Because it requires no enzyme, the approach may be useful in intact cells or tissues, although this remains to be determined.
The manual sorting of thousands of Drosophila embryos necessary to isolate homozygous mutants is both laborious and

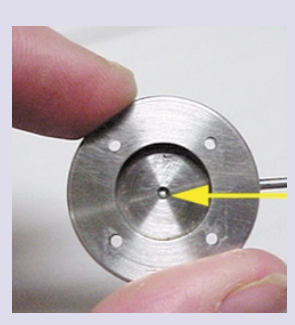
time-consuming. On page 153, Furlong et al. provide a solution,
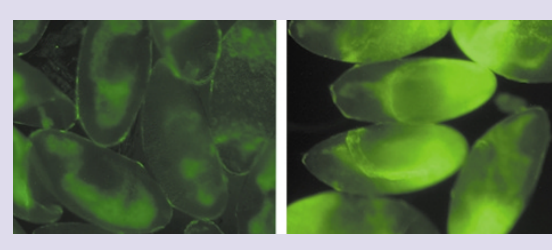
constructing a fluorescence-activated embryo sorter based on embryo expression of green fluorescent protein (GFP). The machine can automatically sort 15 embryos/s with better than 99\% accuracy and has been adapted to sort embryos of other species as well. In addition, Furlong et al. have made available online the detailed plans for their machine. 